Short Communication

\title{
OVERCOMING VITAMIN B12 DEFICIENCY IN VEGAN DIET
}

\author{
Sridevy ${ }^{1} \&$ Prassanna Baby ${ }^{2}$ \\ ${ }^{1}$ Principal Nursing Officer, M other Teresa Post Graduate \& Research Institute of Health Sciences, Pondicherry \\ ${ }^{2}$ Principal, College of Nursing, Sri Ramachandra University, Porur, Chennai, Tamilnadu, INDIA \\ Correspondence \\ Sridevy \\ Principal Nursing Officer, M other Teresa Post Graduate \& Research Institute of Health Sciences, Pondicherry -605006 \\ E-mail : s.sridevy@ rediffmail.com
}

\begin{abstract}
:
Vitamin B12 is a crystalline compound essential to a number of micro-organisms and animals, including humans. So far as is known it is not present in higher plants. Pure vegetarian food is nearly free from vit. B12.It is a nutrient that needs attention in vegan diet. Vegetarians are at risk for vitamin B(12) (B12) deficiency due to suboptimal intake. Dietary deficiency of vitamin B12 due to vegetarianism is increasing and causes hyperhomocysteinemia Areas for research include intermittent vitamin B12 supplement dosing and better measurements of the bioavailability of B12 in fermented vegetarian foods and algae. The goal of the present literature review was to create an awareness among the vegans to identify the vegetarian sources of Vitamin B12 and to incorporate them into their daily diet.
\end{abstract}

Keywords: vegan, fortified sources, macrocytic anemia, perinicious anaemia.

\section{Introduction:}

Vitamin B12 is one of the important elements of the B complex vitamin family. Since the deficiency of this vitamin may result in serious health threats, adequate quantity of intake of this vitamin is essential. Vitamin B12 deficiency disorders are commonly seen only among pure vegetarians, since this vitamin is present only in foods of animal origin. Hence in this article, an attempt has been made to create an awareness among the vegans to identify the vegetarian sources of Vitamin B12 and to incorporate them into their daily diet to overcome the health problems owing to the deficiency of this vitamin.

\section{Importance of Vitamin B12 intake in our daily life}

- The main functions of Vitamin B12 are cell division and production of RBCs.

- Vitamin B12 is essential during pregnancy and lactation.

- Vitamin B12 helps in

Access this article online Quick Response Code

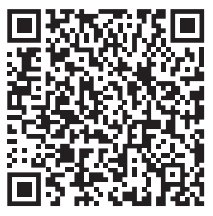
digestion and absorption of nutrients.

- Vitamin B12 plays a vital role in regulating the level of serotonin, a neurotransmitter that maintains the euphoric mood, thereby cures depression in old age.

- It also helps in the secretion of a hormone, melatonin, which stimulatessleep.

- In children, giving oral or parenteral supplements of vitamin B12 may help in relieving wheezing attacks of children suffering from bronchial asthma.

- Vitamin B 12 is produced by the action of bacteria. Plant foods lack vitamin B12 in general. The recommended daily allowance of vitammin B12 is 2.4 micrograms.

\section{Vitamin B12 or Cobalamin deficiency}

Even though the RDA of vitamin B12 is very less, this deficiency leads to many dangerous health threats. The manifestations of this deficiency of vitamin B12 are presented in various ways. The problems include irreversible nerve damage, macrocytic anemia (due to the production of large and immature red blood cells) and pernicious anemia (an inherited disease). In pernicious anemia, the absorption of vitamin B12 is lacking due to the absence of intrinsic factor. Hence those patients have to be supplemented with injection of vitamin B12.

Other serious deficiency disorder is paresthesia ( loss of sensation of the upper and lower extremities), impaired gait, lethargy, excessive tiredness, irregular menstruation, psychological problems like mood swings, depression, 
memory changes, irritability and dementia in elderly.

Deficiency of vitamin B12 leads to increase the level of homocysteine, an amino acid, which increases the risk of CVA and cardiac disorders. High homocysteine level also causes depression.

\section{Causes of poor Vitamin B12 absorption}

I Improper selection of food items, which lack vitamin B12.

I Absence of intrinsic factor in the stomach due to gastritis, partial gastrectomy, ageing process, etc.,

I lleitis, ileal resection.

\section{Sources of Vitamin B12}

Vitamin b12 can be best obtained from animal sources like fish, eggs, dairy products, meat, poultry etc., Plant foods do not contain any reliable and enough vitamin B12 as such. Hence pure vegetarians should identify foods fortified with vitaminB12 and include in their diet or have to depend on the supplements of vitamin B12 to overcome the vitamin B12 deficiency.

I Soy products that are fermented like miso, tempeh, shiitake (dry mushrooms), spirulina and nori (algae) which though actually contain less true vitamin B12, but

\section{References}

1. Institute of Medicine, Food and Nutrition Board: Dietary Reference Intakes for Thiamin, Riboflavin, Niacin, Vitamin B-6, Folate, Vitamin B12, Pantothenic Acid, Biotin, and Choline. Washington, DC: National Academy press, 1998.

2. "Vitamin B12" The American Society of Health-System Pharmacists.

3. Gilligan M (2002). "Metformin and vitamin B12 deficiency". Arch Intern M ed 162 (4): 484-5

4. Eschenmoser A, Wintner CE (June 1977). "Natural product synthesis and vitamin B12". Science 196 (4297): 1410-20. fortified with more analogues of B12.

I Ready to eat, fortified Breakfast cereals like Kellogs corn flakes, Grapenut cereals etc., contain vitamin B12.

I Soy milk, soy beverages, meat analogues (food products which mimic meat, chicken or fish in flavor, texture and appearance, made from wheat gluten or soyabeans) contain fortified vitamin B12.

I Some foods like cheddar cheese, veggie burgers, sunflower margarine, yeast extracts, vegetable stock, sausage mixes and vegetable margarine etc., contain fortified vitamin B12.

In the supermarkets of modern cities, fruits and vegetables are displayed in sanitized manner, after washing with chlorine. Hence vitamin B12 is being removed totally. Hence vegans must incorporate fortified vitamin B12 sources or supplements of vitB12 to meet their RDA of vitamin B12.

\section{Supplements of vitamin B12}

Multivitamin tablets containing vitamin B12 or oral B12 supplement of 5 micrograms is to prescribed meet the daily needs. Intramuscular injection of vitaminB12 can be given if gastric absorption is impaired. 